\title{
Instantaneous ionospheric global conductance maps during an isolated substorm
}

\author{
A. Aksnes ${ }^{1}$, J. Stadsnes ${ }^{1}$, J. Bjordal ${ }^{1, *}$, N. Østgaard ${ }^{2,3}$, R. R. Vondrak ${ }^{2}$, D. L. Detrick ${ }^{4}$, T. J. Rosenberg ${ }^{4}$, \\ G. A. Germany ${ }^{5}$, and D. Chenette ${ }^{6}$ \\ ${ }^{1}$ Department of Physics, University of Bergen, Bergen, Norway \\ ${ }^{2}$ NASA/Goddard Space Flight Center, Greenbelt, MD 20771, USA \\ ${ }^{3}$ University of California, Berkeley, CA 94720-7450, USA \\ ${ }^{4}$ University of Maryland, College Park, USA \\ ${ }^{5}$ University of Alabama in Huntsville, USA \\ ${ }^{6}$ Lockheed Martin Advanced Technology Center, Palo Alto, USA \\ * deceased 17 July 2001
}

Received: 30 November 2001 - Revised: 21 March 2002 - Accepted: 25 March 2002

\begin{abstract}
Data from the Polar Ionospheric X-ray Imager (PIXIE) and the Ultraviolet Imager (UVI) on board the Polar satellite have been used to provide instantaneous global conductance maps. In this study, we focus on an isolated substorm event occurring on 31 July 1997. From the PIXIE and the UVI measurements, the energy spectrum of the precipitating electrons can be derived. By using a model of the upper atmosphere, the resulting conductivity values are generated. We present global maps of how the 5 min time-

electron energies, the UVI electron energy range is more or less comparable with the energy ranges of the statistical models. By calculating the conductivities from combined PIXIE and UVI measurements to compare with the conductivities from using UVI data only, we observe significant differences in the Hall conductance. The greatest differences are observed in the early evening and the late morning sector. We therefore suggest that the existing statistical models underestimate the Hall conductance.
\end{abstract} averaged height-integrated Hall and Pedersen conductivities vary every $15 \mathrm{~min}$ during this isolated substorm. The method presented here enables us to study the time development of the conductivities, with a spatial resolution of $\sim 700 \mathrm{~km}$. During the substorm, a single region of enhanced Hall conductance is observed. The Hall conductance maximum remains situated between latitudes 64 and 70 corrected geomagnetic (CGM) degrees and moves eastward. The strongest conductances are observed in the pre-midnight sector at the start of the substorm expansion. Toward the end of the substorm expansion and into the recovery phase, we find the Hall conductance maximum in the dawn region. We also observe that the Hall to Pedersen conductance ratio for the regions of maximum Hall conductance is increasing throughout the event, indicating a hardening of the electron spectrum. By combining PIXIE and UVI measurements with an assumed energy distribution, we can cover the whole electron energy range responsible for the conductances. Electrons with energies contributing most to the Pedersen conductance are well covered by UVI while PIXIE captures the high energetic component of the precipitating electrons affecting the Hall conductance. Most statistical conductance models have derived conductivities from electron precipitation data below approximately $30 \mathrm{keV}$. Since the intensity of the shortest UVI-wavelengths (LBHS) decreases significantly at higher

Correspondence to: A. Aksnes (arve.aksnes@fi.uib.no)
Key words. Ionosphere (auroral ionosphere, particle precipitation) - Magnetospheric physics (storms and substorms)

\section{Introduction}

Electron precipitation increases the ionization in the Earth's atmosphere, resulting in greater electron densities. This affects the Hall and Pedersen conductivities in the ionosphere, as there is almost a linear relationship between the electrical conductivity and the electron density (Chapman, 1956). During substorms, the electron precipitation varies rapidly. As a result, large changes are occurring in the Hall and Pedersen conductivities.

Below about $150 \mathrm{~km}$, collisions of charged particles with neutrals alter the differential motions of the electrons and the ions. As electrons and ions start to act differently in this height region, we have a total ionospheric electric current density flowing. Throughout the entire conducting ionospheric layer, the electrons are practically tied to the magnetic field as the electron collision frequency is lower than the electron gyrofrequency for heights greater than about $75 \mathrm{~km}$. However, ions are heavily affected by collisions, since the ion collision frequency is greater than the ion gyrofrequency for heights lower than about $125 \mathrm{~km}$. The ionospheric current is traditionally divided into a Pedersen cur- 
rent aligned in the direction of the electric field and a Hall current in the $-\boldsymbol{E} \times \boldsymbol{B}$ direction. The Pedersen conductivity, associated with the Pedersen current, is greatest around $125 \mathrm{~km}$ where the ion collision frequency is comparable with the ion gyrofrequency. The Hall conductivity is greatest below $110 \mathrm{~km}$ where the ions are strongly coupled to the neutrals. The energy of the precipitating electrons is a critical parameter determining the conductivities, as there is a non-linear relation between the energy of a particle and the heights of energy deposition. According to Rees (1963), electrons with energies of about $7 \mathrm{keV}$ or more will deposit most of their energy at heights lower than $110 \mathrm{~km}$ and they will therefore contribute mainly to the Hall conductance. The Pedersen conductance will be most affected by electrons with energies in the energy range of a few $\mathrm{keV}$.

In order to understand the electrodynamic coupling between the ionosphere and the magnetosphere during substorms, we need global conductance distributions. Several studies have generated global models of conductance based on electron precipitation measurements.

Wallis and Budzinski (1981) used electron precipitation data from the International Satellites for Ionospheric Studies (ISIS) 2 satellite. Average electron fluxes with energies of $0.15,1.27,9.65$ and $>22 \mathrm{keV}$ were obtained to calculate the conductivities. Global models of the Hall and Pedersen conductances were developed for two $K_{p}$ ranges, $K_{p} \leq 3$ and $3<K p$. Spiro et al. (1982) determined global values of the Hall and Pedersen conductances for different levels of the $A E$ index. They used electron precipitation data from the Atmospheric Explorer (AE)-C and AE-D satellites, where particle detectors measured electron energies between 0.2 and $27 \mathrm{keV}$. Hardy et al. (1987) presented global maps of the conductances for seven levels of $K_{p}$, ranging from 0 to $\geq 6-$. They used electron precipitation data from the Defence Meteorological Satellite Program (DMSP) F2 and F4, as well as the P78-1 satellite. The particle detectors covered an electron energy range from 0.05 to $20 \mathrm{keV}$. A similar study was performed by Fuller-Rowell and Evans (1987), who inferred height-integrated Hall and Pedersen conductivity values from electron and ion measurements detected between 0.3 and $20 \mathrm{keV}$, using particle precipitation data from the National Oceanic and Atmospheric Administration (NOAA) 6 and 7 satellites. A different approach was made by Ahn et al. (1983); Hall and Pedersen conductances deduced from Chatanika radar data were compared with measurements of the horizontal component of the disturbed magnetic field $(\mathrm{dH})$ measured at College, Alaska. Ahn et al. established empirical relationships between conductances and $d H$ and these were used to compile global conductance models, on the basis of the global distribution of $d H$. A similar but improved ionospheric conductance model by Ahn et al. (1998) took into account different combinations of horizontal $(\mathrm{dH})$ and vertical $(d Z)$ magnetic perturbations to deduce the conductance distribution.

All the conductance models introduced so far organize the results according to the magnetic local time (MLT) sector and activity level. Gjerloev and Hoffman (2000b) used a differ- ent method of organization. A substorm was divided into six different sectors, according to the generic bulge-type auroral substorm defined by Fujii et al. (1994). Each sector had its own characteristic emission and electrodynamical signature. From the Dynamic Explorer (DE) 2 satellite, electron precipitation in the energy range between 0.005 and $32 \mathrm{keV}$, was detected during time periods with auroral substorms. The electron precipitation data from $31 \mathrm{DE} 2$ substorm crossings were sorted and organized with respect to the six substorm sectors instead of sorting by MLT to avoid mixing of data. This was done by comparing the electron measurements with simultaneously obtained auroral images taken by the DE 1 satellite. Finally, these measurements were used to establish a conductance model covering the bulge region. The model of Gjerloev and Hoffman (2000b) is valid for the substorm expansion phase.

Lummerzheim et al. (1991) produced global conductance patterns from instantaneous ultraviolet (UV) images, rather than statistical distributions using auroral images from the DE 1 satellite. By comparing auroral emissions at UV wavelengths, using filter $123 \mathrm{~W}$ and emissions at $557.7 \mathrm{~nm}$, the characteristic energy and flux of the incoming particles were derived. Lummerzheim et al. then used a method developed by Rees et al. (1988) to convert the energy spectra to conductance values. Lummerzheim et al. (1991) did not provide any exact electron energy range; they presented a plot showing the count rate per $\mathrm{erg} \mathrm{cm}^{-2} \mathrm{~s}^{-1}$ energy flux of the $123 \mathrm{~W}$ filter used to deduce the electron spectra. This count rate was plotted as a function of the characteristic energy of the precipitation and it showed a significant decrease with higher characteristic energies. At $10 \mathrm{keV}$, the count rate dropped to 0.01 of the original value, indicating less sensitivity for higher electron energies. This lack of inclusion of high energy electrons is a common limitation among all the conductance models presented. Wallis and Budzinski (1981) do measure electrons above $>22 \mathrm{keV}$ but their data does not provide them with a proper spectral distribution. The best electron energy coverage and spectral calculation is probably done by Gjerloev and Hoffman (2000b), detecting electrons with energies up to $32 \mathrm{keV}$. Nevertheless, even Gjerloev and Hoffman are not able to capture in full the high energy tail of the auroral spectrum which is reported in several papers (Meng et al., 1979; Goldberg et al., 1982; Miller and Vondrak, 1985; Østgaard et al., 2000). In this paper, we will show that these hard spectra have a significant influence on determining the correct value of the Hall conductance.

The conductance model by Ahn et al. (1989) took into account the high energy electron contribution, as they used image data from the DMSP F6 bremsstrahlung X-ray spectrometer instrument. This provided them with an upper electron energy limit of $\sim 70 \mathrm{keV}$, giving good coverage of the electrons contributing to the Hall conductance. However, the lower limit of $\sim 1.5 \mathrm{keV}$ meant that the determination of the Pedersen conductance was rather uncertain, since this conductivity is most influenced by the softer electron energies. Ahn et al. (1989) presented a quasi-instantaneous model. Due to a limited field of view, they could not measure the 

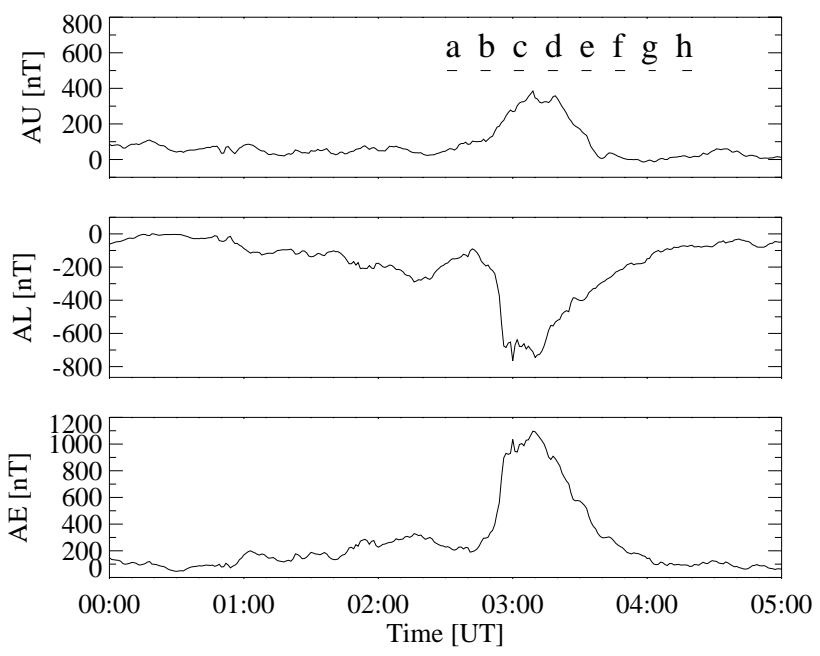

Fig. 1. The $A E, A L$ and $A U$-indices of 31 July 1997. The letters $\mathrm{a}-\mathrm{h}$ correspond to the time periods when we have measurements from PIXIE and UVI.

$\mathrm{X}$-ray distribution over the entire polar region at the same time. It took about $17 \mathrm{~min}$ for the DMSP X-ray spectrometer to make a complete image. They also had to use interpolation routines to fill spatial data gaps in the coverage of the instrument.

The X-ray camera PIXIE and the UV imager UVI onboard the Polar satellite provide global information about the electron precipitation and how it varies both in time and space. We can derive the precipitating electron spectrum by analyzing the UV-emissions and the X-rays. The UVI camera is most sensitive to the softer electron energies. Electron spectra derived from UV emissions will include all electrons responsible for the Pedersen conductance, while the estimation of a proper Hall conductance is more uncertain. A similar but opposite scheme is valid for the PIXIE camera. Electron spectra derived from X-ray measurements provide good coverage of the higher electron energies contributing to the Hall conductance. The estimation of a proper Pedersen conductance is more problematic as PIXIE cannot measure $\mathrm{X}$-rays produced by electrons lower than a few keV. By combining both PIXIE and UVI measurements we can, however, cover the whole electron energy range between about 0.1 and $100 \mathrm{keV}$, as pointed out by Østgaard et al. (2001). We are therefore able to capture also the high-energy tail of the auroral electron spectrum.

In this paper, we make use of X-ray and UV-emission measurements of an isolated substorm event, occurring, approximately, between 02:45 and 04:00 UT on 31 July 1997, to derive instantaneous global conductance maps. The $A E$ index, which we present in Fig. 1, gives indications of a rather intense substorm as the values exceed $1000 \mathrm{nT}$ around 03:00 UT. This substorm occurred in the beginning of a small magnetic storm, although the $D_{s t}$ index only decreased from 14 to $-20 \mathrm{nT}$ during a $3 \mathrm{~h}$ period. The letters a-h correspond to the time periods when we have measurements from PIXIE and UVI.

\section{The procedure: From X-ray and UV-emissions to con- ductance values}

\subsection{From X-ray measurements to electron spectra}

PIXIE is a pinhole camera detecting X-ray photons (Imhof et al., 1995). The useful energy range of these photons is approximately between 2 and $22 \mathrm{keV}$.

A technique for deriving a four-parameter electron spectral distribution from the measured X-ray spectra is described in Østgaard et al. (2000). We only present a brief description here.

To establish the electron spectra causing the measured Xrays, we make use of a look-up table provided by a coupled electron photon transport code originally derived from neutron transport codes (Lorence, 1992). This look-up table gives us the X-ray production emitted at different zenith angles for electrons with different exponential energy spectra. Isotropic electron precipitation has been assumed.

As described by Østgaard et al. (2001), the X-ray measurements are divided into 120 boxes. A box is 6 corrected geomagnetic (CGM) degrees latitude by $1 \mathrm{~h}$ MLT, starting at CGM latitude $52^{\circ}$ until $82^{\circ}$. From the X-ray spectrum measured in each box, we derive a corresponding electron spectrum by using the look-up table as described above.

\subsection{From UV emission measurements to electron spectra}

We will give a short description of the method used to derive the average electron energy and the electron energy flux from UV-emissions measured by the UVI camera. For a more detailed description, see Germany et al. (1997, 1998a, b).

Global images of emissions within the Lyman-BirgeHopfield (LBH) band $(140-180 \mathrm{~nm})$ are provided by the UVI instrument (Torr et al., 1995). The LBH-emissions are divided into LBHS (140-160 nm) and LBHL (160$180 \mathrm{~nm}$ ) respectively. Within the UVI band pass, the $\mathrm{O}_{2}$ Schumann-Runge absorption continuum peaks at the shorter wavelengths and decreases with longer wavelengths. Thus, the shorter wavelengths (LBHS) are subject to significantly greater $\mathrm{O}_{2}$ absorption than the longer wavelengths (LBHL).

The height level for production of UV emissions depends on the incoming electron energy. Electrons of higher energies deposit their energy deeper in the atmosphere, resulting in a stronger absorption of the UV-emissions as they propagate out of the atmosphere.

The average electron energy is derived from the ratio of the intensities of the two LBH bands using an assumed spectral shape. Similarly, the energy flux is derived from the LBHL intensity.

\subsection{Electron spectra derived from both PIXIE and UVI measurements}

We use the average electron energy and the electron energy flux from UVI measurements to fit an exponential as well as a Maxwellian distribution. The final choice of spectrum for the lower electron energy range depends on which one gives 
Table 1. Maximum Hall and Pedersen conductance in magnitude and location for the isolated substorm event on 31 July 1997 , as derived from the combined PIXIE and UVI measurements as well as from UVI data only. We also present the differences in percent between the conductance values using electron spectra derived from both PIXIE and UVI compared with the values obtained using spectra derived from UVI only. The letters a-h correspond to the 8 time periods as given in Fig. 2

\begin{tabular}{|c|c|c|c|c|c|c|c|}
\hline \multirow[b]{2}{*}{$\begin{array}{l}\text { Time } \\
\text { Period }\end{array}$} & \multirow[b]{2}{*}{$\begin{array}{l}\text { MLT } \\
\text { Sector }\end{array}$} & \multicolumn{3}{|c|}{ Hall conductance } & \multicolumn{3}{|c|}{ Pedersen conductance } \\
\hline & & $\begin{array}{c}\text { UVI } \\
{[S]}\end{array}$ & $\begin{array}{c}\text { PIXIE } \\
+ \text { UVI } \\
{[\mathrm{S}]}\end{array}$ & $\begin{array}{c}\text { Difference: } \\
\text { UVI vs } \\
\text { PIXIE + UVI } \\
{[\%]}\end{array}$ & $\begin{array}{l}\text { UVI } \\
{[\mathrm{S}]}\end{array}$ & $\begin{array}{c}\text { PIXIE } \\
+ \text { UVI } \\
\quad[\mathrm{S}]\end{array}$ & $\begin{array}{c}\text { Difference: } \\
\text { UVI vs } \\
\text { PIXIE + UVI } \\
{[\%]}\end{array}$ \\
\hline$(\mathrm{a}) *$ & $20: 00-21: 00$ & 28 & 27 & -3.6 & 15 & 15 & 0.0 \\
\hline (b) & 23:00-00:00 & 47 & 53 & 13 & 25 & 25 & 0.0 \\
\hline (c) & 01:00-02:00 & 41 & 51 & 24 & 22 & 22 & 0.0 \\
\hline (d) & 03:00-04:00 & 24 & 29 & 21 & 12 & 12 & 0.0 \\
\hline \multirow[t]{2}{*}{ (e) } & 01:00-02:00 & 17 & 23 & 35 & 7.6 & 7.8 & 2.6 \\
\hline & 05:00-06:00 & 19 & 23 & 21 & 8.7 & 8.9 & 2.3 \\
\hline (f) & 08:00-09:00 & 16 & 21 & 31 & 7.8 & 8.0 & 2.6 \\
\hline \multirow[t]{2}{*}{ (g) } & 08:00-09:00 & 18 & 20 & 11 & 7.7 & 7.7 & 0.0 \\
\hline & 09:00-10:00 & 15 & 20 & 33 & 6.6 & 6.8 & 3.0 \\
\hline \multirow[t]{2}{*}{ (h) } & 06:00-07:00 & 12 & 16 & 33 & 5.3 & 5.5 & 3.8 \\
\hline & 08:00-09:00 & 12 & 16 & 33 & 5.8 & 6.0 & 3.4 \\
\hline
\end{tabular}

\footnotetext{
* Note that in our method the upper energy limit of the UVI spectrum is lower when merged with the PIXIE spectrum than is its value when UVI is used alone. As a result, under some circumstances, for example, during the time period 02:30:40-02:35:10 UT (row (a)), larger conductances can be obtained from the UVI only spectrum than from the UVI plus PIXIE spectrum.
}

the smoothest transition to the exponential electron spectrum derived from the measured X-ray spectrum.

For this particular event, 31 July 1997, Østgaard et al. (2001) have compared measured electron spectra, from the polar orbiting satellites DMSP F14 and NOAA 12, with derived electron spectra from the PIXIE and UVI measurements. Regarding the energy flux between $90 \mathrm{eV}$ and $30 \mathrm{keV}$, they find an average ratio of $1.03 \pm 0.6$ by comparing measured and derived electron spectra, indicating good agreement. The polar orbiting satellites measure the electron fluxes, only along a line through the large pixels of about $700 \mathrm{~km} \times 700 \mathrm{~km}$ in the PIXIE measurements. Thus the electron fluxes measured by these satellites are not necessarily the same as the average electron flux in the corresponding PIXIE pixel. This explains the large standard deviation.

2.4 Deriving the conductance values from the energy spectra

We use a computer code developed by University of Maryland based on the TANGLE code (for more details on TANGLE, see Vondrak and Baron, 1976; Vondrak and Robinson, 1985). The code models the ionospheric effects of energy deposition into the atmosphere by energetic particles. One possible output is conductivity. Input is the electron spectrum as derived from PIXIE and UVI measurements. To model the atmosphere, the program code uses the Mass Spectrometer and Incoherent Scatter Extended Atmospheric model (MSISE-90). The electron energy deposition function is the cosinedependent Isotropic over the Downward Hemisphere (IDH) model of Rees (1963). Rees (1989) has also contributed an expression for the electron range function.

First, the electron production $q$ from the precipitation is calculated. We should point out that $q$ does not include any contribution from the solar illumination. The dayglow has been removed from the UVI images and the background originating from other sources than particle precipitation has been removed from the PIXIE data. To establish a value of the total electron density $N_{e}$, we therefore have to include a background electron source term $q_{0}$ in the time-dependent rate equation:

$d N_{e} / d t=q(h)+q_{0}(h)-\alpha_{e f f}(h) * N_{e}^{2}(h)$

$q_{0}$ is taken from the International Reference Ionosphere (IRI)-95 model, which is an empirical standard model of the ionosphere. $\alpha_{e f f}$ is the effective dissociative recombination coefficient. The program uses formulas derived from Vickrey et al. (1982) and Gledhill (1986) to calculate $\alpha_{e f f}$. We should point out that we have not included diffusive transport in the equation as this is negligible at the heights contributing to the conductances. Chemical equilibrium is assumed, since the recombination time is on the order of seconds. We therefore set $d N_{e} / d t=0$.

Finally the code derives the conductivities, using ionneutral collision frequencies from Vickrey et al. (1981) and electron-neutral collision frequencies from Thrane and Piggott (1966). 
Table 2. Maximum Hall and Pedersen conductance in magnitude and location for five statistical conductance models

\begin{tabular}{lcccccc}
\hline Model & MLT & ILAT & Hall & Pedersen & Ratio H/P & Activity level \\
\hline Wallis and & $21: 00$ & 68 & 19 & 12 & 1.6 & $3<K_{p}<=9$ \\
Budzinski (1981) & $05: 00$ & 65 & 27 & 13 & 2.1 & \\
Fuller-Rowell and & 20:00-23:00 & 65 & 23 & 16 & 1.4 & $K_{p}=5-$ \\
Evans (1987) & & & & & & \\
Hardy et al. (1987) & $00: 00-00: 30$ & $66^{*}$ & 17 & 8.6 & 2.0 & $K_{p}=4$ \\
& $04: 30-05: 00$ & $66^{*}$ & 20 & 8.0 & 2.5 & \\
Spiro et al. (1982) & $18: 00-19: 00$ & 72 & 34 & 15 & 2.3 & $A E>600$ nT \\
& $21: 00-22: 00$ & 74 & 34 & 15 & 2.3 & \\
& $04: 00-05: 00$ & 65 & 29 & 13 & 2.2 & \\
Gjerloev and & $21: 00$ & 69 & 48 & 20 & 2.4 & Substorm \\
Hoffman (2000b) & $01: 30$ & 65 & 22 & 10 & 2.2 & \\
\hline
\end{tabular}

* Note that the model of Hardy et al. (1987) uses CGM latitudes. The other models give the position in invariant latitude (ILAT).

\section{Results}

In Fig. 2, we present global maps of how the conductances derived from combined PIXIE and UVI measurements vary during this isolated substorm. A new X-ray image is produced from the PIXIE measurements every $15 \mathrm{~min}$ with an integration time of about $4.5 \mathrm{~min}$. UVI images were selected to match the PIXIE observations. This procedure has been explained by Østgaard et al. (2001) in detail. Østgaard et al. (2000) have analyzed the electron precipitation during this event, and they have shown that substorm onset occurs at 02:40:30 UT in the MLT sector 20:00-21:00. We have derived the global distribution of conductance and we have images showing the conductances before substorm onset (Fig. 2a), during the substorm expansion and recovery phase (Fig. 2b-g) and until the end of recovery phase (Fig. 2h). Also presented in Fig. 2 are global maps of Hall to Pedersen conductance ratios. We require the Pedersen conductance to be greater than $4 \mathrm{~s}$ in order to calculate a Hall to Pedersen conductance ratio.

Our results give the strongest conductances in the substorm onset region in the pre-midnight sector (Fig. 2b). Here we find a maximum Hall conductance value of $53 \mathrm{~s}$ and a maximum Pedersen conductance of $25 \mathrm{~s}$, as presented in Table 1 .

By looking at the successive conductance maps in Fig. 2, we find that the Hall conductance maximum remains situated between CGM latitudes $64-70^{\circ}$ during the whole substorm event. The image before substorm onset (Fig. 2a) places the Hall conductance maximum in the MLT sector 20:00-21:00. This maximum moves eastward with every subsequent data set, as presented in Table 1.

The scatter in Fig. 2 may reflect the uncertainty in deriving proper electron energy spectra. Boxes marked with a black dot indicate that we have only included the background electron density when deriving the conductances although there may be some weak precipitation.

We have calculated the conductance values using electron spectra derived from both PIXIE and UVI; we compared these with the values obtained using spectra derived from UVI only. This is done in order to examine how sensitive the conductance values are to the energetic tail in the energy spectra. The study is motivated by the fact that most of the statistical conductance models presented are based on measurements of precipitating electrons at energies below approximately $30 \mathrm{keV}$. Since the UVI LBHS intensity decreases significantly at higher electron energies, the UVI electron energy range is more or less comparable with the energy ranges of most of the statistical models. Therefore, in Table 1, we have also included conductance values derived from UVI measurements only. We find that combining PIXIE and UVI results in significantly greater Hall conductance values. This is due to the fact that PIXIE captures the high energy tail of the precipitating electron energy spectra while UVI measurements do not provide sufficient information to derive the spectral shape of these high electron energies. We find only minor differences for the Pedersen conductance. This was to be expected, as UVI measures well the softer electron energies which are most significant to the Pedersen conductance. The differences between conductance values deduced from both PIXIE and UVI measurements and conductance values from using UVI only have been studied more closely. In Fig. 3, we present such differences for six MLT sectors. Note that we are looking at data from CGM latitudes $64-70^{\circ}$, since this is the area of maximum conductance (see Fig. 2). We have further disregarded data when the Hall conductances, estimated from the UVI measurements, are lower than $7 \mathrm{~s}$, in order to avoid using uncertain conductance values. The mean difference in the Hall conductance estimated for MLT sectors 18:00-21:00 is $\sim 36 \%$. This means that the Hall conductances derived when combining PIXIE and UVI are about $36 \%$ greater than the values derived from UVI data only. In the next MLT sector, between 21:00-24:00, we find a somewhat lower mean difference of about $21 \%$ in the Hall conductances. Thereafter, this mean value exceeds $\sim 24, \sim 26$ and $\sim 29 \%$, respectively, for the MLT sectors 00:00-03:00, 


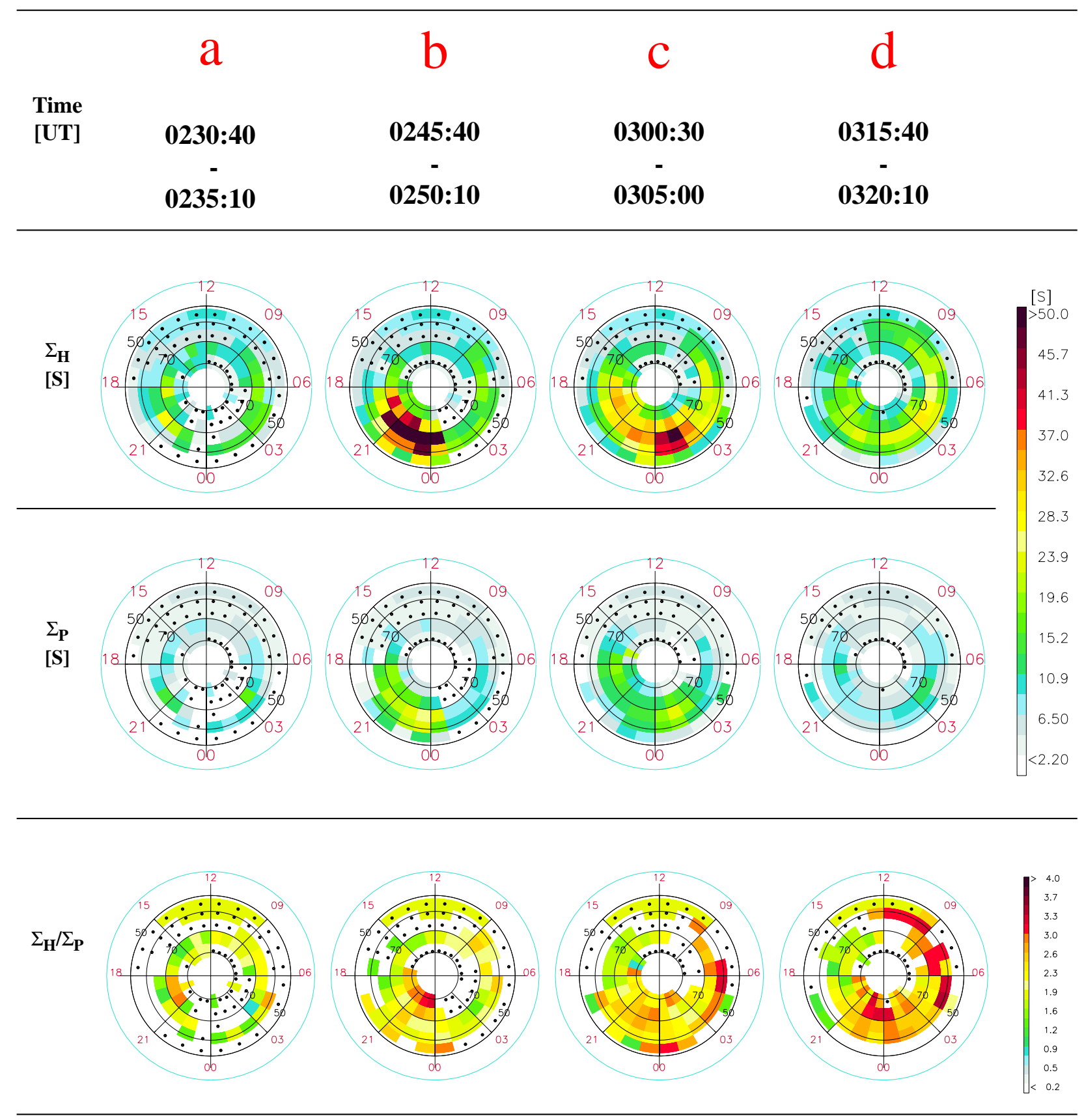

Fig. 2. Polar plots of the Hall and Pedersen conductances for 8 successive time periods during the isolated substorm event on 31 July 1997 . Boxes where we have only included the contribution to the conductances from the background are marked with a black dot. Also presented are the Hall to Pedersen conductance ratios.

03:00-06:00 and 06:00-09:00. A mean difference in the Hall conductance value of $\sim 48 \%$ for the last MLT sector between 09:00 and 12:00 indicates a hardening of the electron spectra on the morningside.

\section{Discussion}

A comparison between our instantaneous global conductance maps and the statistical models has been done. In order to do such a comparison, we make use of the $A E$ and $K_{p}$ indices. Except for the model by Gjerloev and Hoffman (2000b), all the statistical models presented depend strongly on either the value of the $A E$ index or the $K_{p}$ index. Usually, the $A E$ index should be the best correlation parameter. While $K_{p}$ is a $3 \mathrm{~h}$ index, $A E$ can be estimated every minute. However, for this event on 31 July 1997, only four geomagnetic stations have contributed to the provisional $A E$ index. This implies a probability that electrons may precipitate in a region not 


\begin{tabular}{ccccc} 
& e & $f$ & $g$ & h \\
Time & & & & \\
{$[$ UT $]$} & $0330: 40$ & $0345: 40$ & $0400: 40$ & $0415: 40$ \\
& - & - & - & - \\
& $0335: 10$ & $0350: 10$ & $0403: 50$ & $0420: 00$ \\
\hline
\end{tabular}
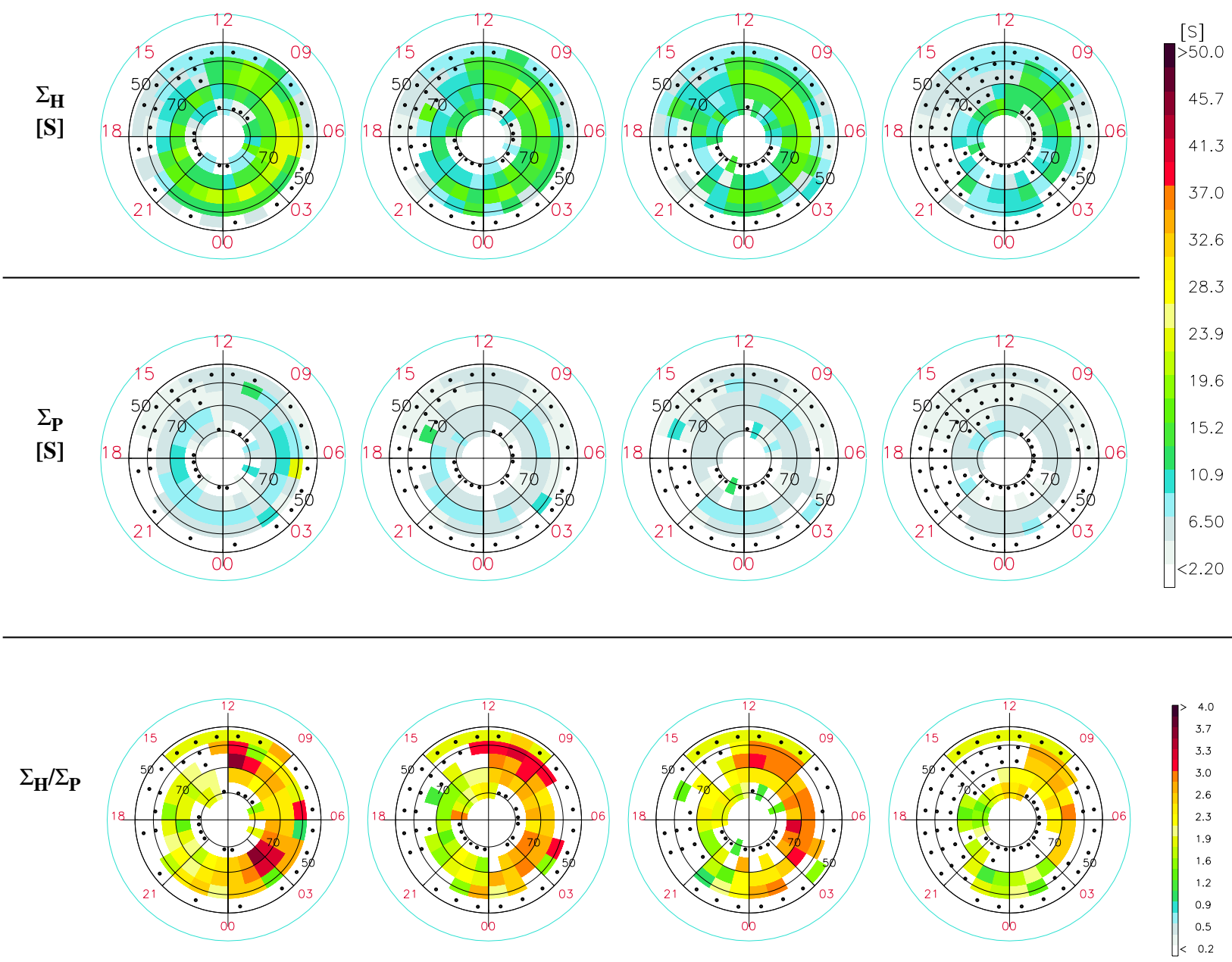

Fig. 2. continued ...

covered by the stations contributing to this $A E$ index. From Fig. 2, we find that the strongest conductances are present in the time period 02:45:40-02:50:10 UT. According to Fig. 1, this takes place while the $A E$ index is still rather low although, by looking at magnetic field data from the Canadian magnetometer station at Fort Churchill located in the region of the precipitation, we observe abrupt decreases in the magnetic field measurements around 02:45 UT. Consequently, we must be cautious when comparing the $A E$ index and the global conductance distribution for this case.

Since we have instantaneous data, we have a tool to study the time development of the conductivities. However, the spatial resolution of the PIXIE camera is a severe restriction, as it is $\sim 700 \mathrm{~km}$. This prevents us from studying small scale features and gradients.

As presented in Table 2, most of the statistical models have several conductance maximum regions. Our instantaneous global conductance maps (Fig. 2) show only one maximum for most of the time periods presented.

In Table 2, we have summarized published Hall to Pedersen conductance ratios. We find that this parameter varies between 1.4 and 2.5 . 
Conductances Derived From PIXIE and UVI vs UVI Only CGM latitudes 64-70 degrees

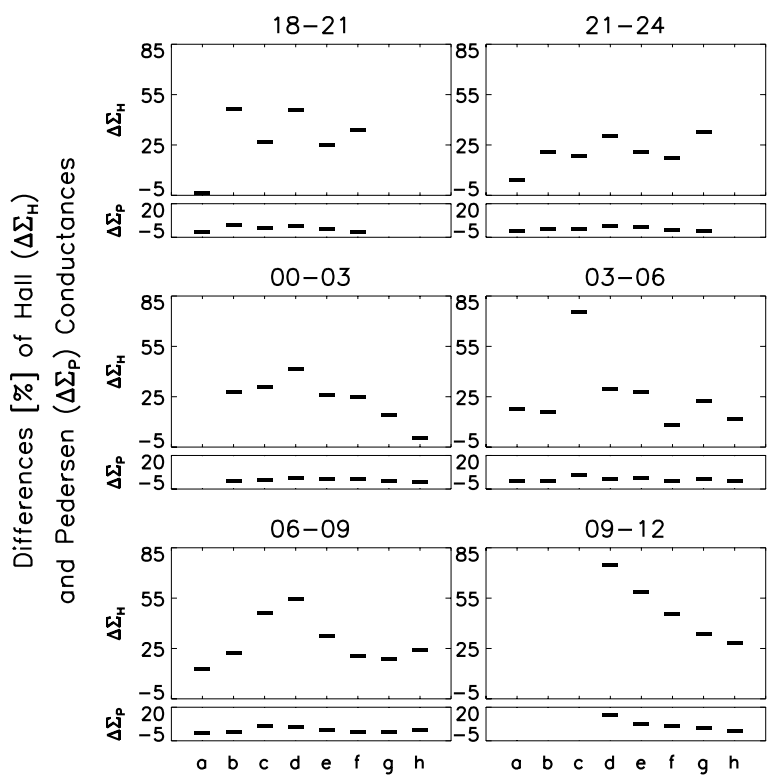

Fig. 3. Differences between conductances derived from the combined PIXIE and UVI measurements and conductances derived from UVI data only. We study six different MLT sectors for CGM latitudes $64-70^{\circ}$. The differences are given in percent. The letters $\mathrm{a}-\mathrm{h}$ correspond to the 8 time periods given in Fig. 2 .

The Hall to Pedersen conductance ratios derived from our measurements are given in Table 3. Ratios are given for conductances derived from combined PIXIE and UVI as well as from UVI only. We study the same regions as those given in Table 1 but now we have not included the contribution of the background density. This is done because all the conductance models (except Wallis and Budzinski, 1981) only take precipitation data into account. It is interesting to note that the ratios derived from using UVI measurements only are in the same range as the ratio values from the different conductance models (Table 2). The ratios derived from combining PIXIE and UVI are on the average $\sim 20 \%$ greater. Since the statistical models in Table 2 operate with almost the same electron energy detection range as the UVI imager, we suggest, therefore, that the statistical models presented probably underestimate the Hall conductance. Consequently, the Hall to Pedersen conductance ratio depends strongly on whether or not we include the PIXIE measurements. This ratio is often used as an indicator of the hardness of the precipitating electrons. For example, Brekke et al. (1989) derived an empirical formula between the root-mean-square energy of the precipitating electrons and the Hall to Pedersen conductance ratio. Germany et al. (1994), using a different approach, found a power law dependence of conductances on the energy flux.

A different kind of underestimation was pointed out by Robinson et al. (1987). They came up with empirical formulae relating Hall and Pedersen conductances to the average
Table 3. The Hall to Pedersen conductance ratios derived from the combined PIXIE and UVI measurements, as well as from UVI data only, for the regions of maximum conductance (given in Table 1). The letters $\mathrm{a}-\mathrm{h}$ correspond to the 8 time periods given in Fig. 2 . Background electron density is not included

\begin{tabular}{lccc}
\hline Time & MLT & $\begin{array}{c}\text { UVI } \\
\text { Radio H/P }\end{array}$ & $\begin{array}{c}\text { PIXIE \& UVI } \\
\text { Radio H/P }\end{array}$ \\
\hline (a) & 20:00-21:00 & 1.9 & 1.8 \\
(b) & $23: 00-00: 00$ & 1.9 & 2.1 \\
(c) & $01: 00-02: 00$ & 1.9 & 2.3 \\
(d) & $03: 00-04: 00$ & 2.1 & 2.4 \\
(e) & $01: 00-02: 00$ & 2.3 & 2.9 \\
& $05: 00-06: 00$ & 2.2 & 2.6 \\
(f) & $08: 00-09: 00$ & 2.1 & 2.7 \\
(g) & $08: 00-09: 00$ & 2.4 & 2.7 \\
& $09: 00-10: 00$ & 2.4 & 3.1 \\
(h) & $06: 00-07: 00$ & 2.4 & 3.0 \\
& $08: 00-09: 00$ & 2.2. & 2.9 \\
\hline
\end{tabular}

energy and energy flux of the electrons. According to Robinson et al. (1987), it is essential to integrate over a broad electron energy range when calculating the mean energy value. However, errors do arise in the application of their equations if too many lower electron energies are included. They suggest a lower limit of $500 \mathrm{eV}$ as secondary electron fluxes become important at energies below. As Spiro et al. (1982) estimated a mean energy value and thereafter the conductivities from an electron detection range of $0.2-27 \mathrm{keV}$, they probably underestimated the conductances in two ways. First of all, they included too many lower electron energies in the calculation of the total number flux. These lower electron energies deposit their energy too high in the atmosphere to affect conductances and should therefore not be included in the calculation of a mean energy value. When the energy flux is divided by the number flux including these low energies, the resulting values of mean energy cannot be used in the empirical relations for conductance. Secondly, they should have extrapolated their spectra to higher energies, according to Robinson et al. (1987). Hardy et al. (1987) took the results of Robinson et al. (1987) into account, as they both introduced a low-energy cutoff of approximately $500 \mathrm{eV}$ as well as an extrapolation of the spectra to $100 \mathrm{keV}$ before estimating the mean energy value. However, Hardy et al. (1987) will not capture the high energy tail, as pointed out in the introduction.

We find that a combination of both PIXIE and UVI measurements is needed in order to cover all electron energies contributing to the conductances. However, our results also underestimate the conductances since the proton precipitation will not contribute to the X-ray measurements. The UVI camera is thought to be partly sensitive to protons also. According to Frey et al. (2001), LBH emission may contain contributions from proton excitation, although this is limited to localized regions with great proton precipitation. The model 
by Fuller-Rowell and Evans (1987) is the only model presented, based on particle detection, which takes into account this contribution. Fuller-Rowell and Evans have included the proton energy flux in their calculation by treating the protons as if they were electrons. According to Galand et al. (2001), electrons are the dominant source of ionization. In a statistical study during moderate magnetic activity $\left(K_{p}=3\right)$, they found that protons contributed $\sim 15 \%$ to the total energy inferred although this is strongly dependent upon location. This result is similar to an earlier study by Hardy et al. (1989), which found that the hemispheric energy input from the ions varied between 11 and 17\% of the energy input for the electrons.

From Table 3, we observe that the Hall to Pedersen conductance ratio for regions of maximum Hall conductance is increasing throughout the event. This indicates a hardening of the electron spectra as the maximum moves to the morningside.

Around substorm onset, at 02:45:40-02:50:10 UT, we find, from Fig. 2, several boxes in the pre-midnight sector showing a strong Hall conductance. From 20:00 to 00:00 MLT, the Hall conductance is between 48 and $53 \mathrm{~s}$. The maximum Hall conductance values in the pre-midnight sector from the previous studies varies between $19 \mathrm{~s}$ (Wallis and Budzinski, 1981), 23 s (Fuller-Rowell and Evans, 1987) and $34 \mathrm{~s}$ (Spiro et al., 1982). A qualitative comparison between such maximum values is difficult. First of all, substorms are highly variable and we compare results from just one substorm event with models based on average values. We should point out that our conductance maximum value is associated with the start of the substorm expansion phase, when energy is loaded into the ionosphere. We also have a significant difference in spatial resolution. Most of the statistical conductance models bin their values in a $1^{\circ}$ latitude grid. When using PIXIE and UVI measurements, we have to include data from six degrees latitude (as pointed out in Sect. 2.1) in order to derive reliable electron spectra and thereafter conductance values. The time resolution is also in favor of the statistical models as the integration time is measured in seconds while PIXIE and UVI estimates require several minutes of data. This leaves us with lower values than a better resolution in both space and time would give us. However, while we have the possibility to present instantaneous values, the statistical models must take into account a great number of measurements. This will result in a smoothing of the conductance values and thereby a reduction compared to the peak values of individual measurements. Finally, as already pointed out, the statistical models probably underestimate the Hall conductance whenever a hard electron spectral component is present.

The statistical model of Gjerloev and Hoffman (2000b) reports a Hall conductance value of $48 \mathrm{~s}$. This is a significantly greater value than the maxima from the other statistical conductance models. By organizing their data according to the electrodynamical signature of the substorm instead of using MLT, they avoid a mixing of data from different parts of the substorm. Further, they have restricted their model of conductances to time periods with auroral substorms. The main reason for the high conductance values of Gjerloev and Hoffman (2000a) is the fine spatial resolution in the measurements of the precipitation events, allowing them to identify Hall conductance peaks exceeding $100 \mathrm{~s}$. Such values are reported to have a typical size of $\sim 20 \mathrm{~km}$ and are associated with energetic $(>10 \mathrm{keV})$ inverted $\mathrm{V}$ events. With our much coarser spatial resolution $(\sim 700 \mathrm{~km})$, we are not able to identify such small scale features by using PIXIE and UVI measurements.

Kirkwood et al. (1988) used the European Incoherent Scatter (EISCAT) radar to examine the conductivity changes during substorms. They selected seven substorm events and measured the electron density profile in order to calculate the conductances. By using the incoherent scatter radar, they could produce electron density profiles with high resolution in both altitude $(2 \mathrm{~km})$ and time $(5 \mathrm{~s})$. In accordance with Gjerloev and Hoffman (2000b), Kirkwood et al. found Hall conductance values exceeding $100 \mathrm{~s}$, the highest Hall and Pedersen conductances reaching $120 \mathrm{~s}$ and $48 \mathrm{~s}$, respectively; they could not, however, present the global conductance distribution as radar measurements are localized to small regions.

We observe that the conductance maximum moves eastward throughout the substorm event. In the later phases of the substorm, the conductance maximum is found on the morningside. This can be associated with the maximum in the energetic electron precipitation which typically develops in the dawn region during substorms (Østgaard et al., 2000). A maximum on the morningside is also seen in several previous studies (Wallis and Budzinski, 1981; Spiro et al., 1982; Hardy et al., 1987).

From Fig. 2, we find that the location of the maximum Hall conductance for all time periods is between CGM latitudes $64-70^{\circ}$. This corresponds well with the different model results, as presented in Table 2.

\section{Conclusion}

By using X-ray data from PIXIE and UV emission data from UVI, we can derive the energy spectrum and the fluxes of the precipitating energetic electrons over a wide energy range. We can then calculate the associated ionization and conductances in the upper atmosphere.

In this paper, we have shown that a combination of PIXIE and UVI data is needed in order to cover the whole electron energy range influencing the conductivities. By deriving conductivities from UVI data only, we find that the Hall conductance is underestimated. This underestimation is strongest in MLT sector 09:00-12:00 for the isolated substorm event of 31 July 1997. Including PIXIE measurements gives Hall conductance values approximately $48 \%$ greater than those deduced from UVI only. Most of the existing statistical models have derived the conductivities by detecting electrons in an energy range comparable with the UVI imager. We therefore suggest that these statistical models underestimate the 
Hall conductance. This is further supported by an analysis of the Hall to Pedersen conductance ratio. From UVI measurements only, we derive values comparable with the model results. However, introducing PIXIE data results in significantly greater Hall to Pedersen conductance ratio values.

In contrast to statistical conductance models, we can study the time development of the conductances throughout a specific substorm event by using PIXIE and UVI measurements. For the isolated substorm event occurring on 31 July 1997, we have generated global maps of the Hall and Pedersen conductances. The strongest conductances are observed in the substorm onset region in the pre-midnight sector. The Hall conductance maximum remains situated between CGM latitudes $64-70^{\circ}$ during the whole substorm event and moves eastward. In the late recovery phase of the substorm, we observe a conductance maximum in the dawn region. The Hall to Pedersen conductance ratio for the locations with maximum Hall conductance is increasing throughout the event. This indicates a hardening of the electron spectra as the conductance maximum moves eastward on the morningside.

Acknowledgements. This study was supported by the Norwegian Research Council (NFR).

The work was also supported by NASA UVI funding from University of California, Berkeley grant SA3216 to the University of Alabama in Huntsville, by contract F49620-00-0-0204 from the Air Force Office of Scientific Research and by NASA grant NAG510743 (G. A. Germany).

Work at the University of Maryland has been supported by NASA contract NAS5-30372 under Subcontract No. SE70A0470R from the Lockheed Martin Advanced Technology Center.

J. Stadsnes thanks the staff of the NASA/GSFC Laboratory for Extraterrestrial Physics for their hospitality and support during his stay in 2000 .

We are grateful to COSPAR and URSI for generating and making available via the Internet, IRI-95 an empirical standard model of the ionosphere.

We are also grateful to the World Data Center - C2 (T. Kamei) in Kyoto, Japan, for making quick look AE indices available via the Internet.

Topical Editor M. Lester thanks R. M. Robinson and another referee for their help in evaluating this paper.

\section{References}

Ahn, B.-H., Robinson, R. M., Kamide, Y., and Akasofu, S.-I.: Electric conductivities, electric fields and auroral particle energy injection rate in the auroral ionosphere and their empirical relations to the horizontal magnetic disturbances, Planet. Space. Sci., 31, $641,1983$.

Ahn, B.-H., Kroehl, H. W., Kamide, Y., and Gorney, D. J.: Estimation of ionospheric electrodynamic parameters using ionospheric conductance deduced from bremsstrahlung $\mathrm{X}$ ray image data, J. Geophys. Res., 94, 2565, 1989.

Ahn, B.-H., Richmond, A. D., Kamide, Y., Kroehl, H. W., Emery, B. A., de la Beaujardiere, O., and Akasofu, S. I.: An ionospheric conductance model based on ground magnetic disturbance data, J. Geophys. Res., 103, 14769, 1998.
Brekke, A., Hall, C., and Hansen, T. L.: Auroral ionospheric conductances during disturbed conditions, Ann. Geophysicae, 7, 269, 1989.

Chapman, S.: The electrical conductivity of the ionosphere: A review, Nuovo Cimento, 4, 1385, 1956.

Frey, H. U., Mende, S. B., Carlson, C. W., Gerard, J.-C., Hubert, B., Spann, J., Gladstone, R., and Immel, T. J.: The electron and proton aurora as seen by IMAGE-FUV and FAST, Geophys. Res. Lett., 28, 1135, 2001.

Fujii, R., Hoffman, R. A., Anderson, P. C., Craven, J. D., Sugiura, M., Frank, L. A., and Maynard, N. C.: Electrodynamical parameters in the nighttime sector during auroral substorms, J. Geophys. Res., 99, 6093, 1994.

Fuller-Rowell, T. J. and Evans, D. S.: Height-integrated Pedersen and Hall conductivity patterns inferred from the TIROS-NOAA satellite data, J. Geophys. Res., 92, 7606, 1987.

Galand, M., Fuller-Rowell, T. J., and Codrescu, M. V.: Response of the upper atmosphere to auroral protons, J. Geophys. Res., 106, 127, 2001.

Germany, G. A., Torr, D. G., Richards, P. G., Torr, M. R., and John, S.: Determination of ionospheric conductivities from FUV auroral emissions , J. Geophys. Res., 99, 23 297, 1994.

Germany, G. A., Parks, G. K., Brittnacher, M., Cumnock, J., Lummerzheim, D., Spann, J. F., Chen, L., Richards, P. G., and Rich, F. J.: Remote determination of auroral energy characteristics during substorm activity, Geophys. Res. Lett., 24, 995, 1997.

Germany, G. A., Parks, G. K., Brittnacher, M., Spann, J. F., Cumnock, J., Lummerzheim, D., Rich, F. J., and Richards, P. G.: Energy characterization of a dynamic auroral event using GGS UVI images, in Geospace Mass and Energy Flow: Results from the International Solar-Terrestrial Physics Program, edited by Horwitz, J. L., Gallagher, D. L., and Peterson, W. K., AGU, Washington, D.C., 143, 1998a.

Germany, G. A., Spann, J. F., Parks, G. K., Brittnacher, M., Elsen, R., Chen, L., Lummerzheim, D., and Rees, M.: Auroral observations from the Polar Ultraviolet Imager (UVI), in Geospace Mass and Energy Flow: Results from the International SolarTerrestrial Physics Program, edited by Horwitz, J. L., Gallagher, D. L., and Peterson, W. K., AGU, Washington, D.C., 149, 1998b.

Gjerloev, J. W. and Hoffman, R. A.: Height-integrated conductivity in auroral substorms. 1. Data, J. Geophys. Res., 105, 215, 2000a.

Gjerloev, J. W. and Hoffman, R. A.: Height-integrated conductivity in auroral substorms. 2. Modelling, J. Geophys. Res., 105, 227, 2000 b.

Gledhill, J. A.: The effective recombination coefficient of electrons in the ionosphere between 50 and $150 \mathrm{~km}$, Radio Sci., 21, 3, 339, 1986.

Goldberg, R. A., Barcus, J. R., Treinish, L. A., and Vondrak, R. R.: Mapping of auroral X rays from rocket overflights, J. Geophys. Res., 87, 2509, 1982.

Hardy, D. A., Gussenhoven, M. S., Vaistrick, R., and McNeil, W. J.: Statistical and functional representations of the pattern of auroral energy flux, number flux, and conductivity, J. Geophys. Res., 92, 12, 275, 1987.

Hardy, D. A., Gussenhoven, M. S., and Brautigam, D.: A Statistical Model of Auroral Ion Precipitation, J. Geophys. Res., 94, 370, 1989.

Imhof, W. L., Spear, K. .A., Hamilton, J. W., Higgins, B. R., Murphy, M. J., Pronko, J. G., Vondrak, R. R., McKenzie, D. L., Rice, C. J., Gorney, D. J., Roux, D. A., Williams, R. L., Stein, J. A., Bjordal, J., Stadsnes, J., Njoten, K., Rosenberg, T. J., Lutz, L., and Detrick, D. L.: The Polar Ionospheric X-ray Imaging Exper- 
iment (PIXIE), Space Sci. Rev., 71, 385, 1995.

Kirkwood, S., Opgenoorth, H., and Murphree, J. S.: Ionospheric conductivities, electric fields and currents associated with auroral substorms measured by the EISCAT radar Planet. Space. Sci., 36, 1359, 1988

Lorence, L. J.: CEPXS/ONELD Version 2.0: A discrete ordinates code package for general one-dimensional coupled electronphoton transport, IEES Trans. Nucl. Sci, 39, 1031, 1992.

Lummerzheim, D., Rees, M. H., Craven, J. D., and Frank, L. A.: Ionospheric conductances derived from DE-1 auroral images, J. atmos. terr. Phys., 53, 281, 1991.

Meng, C.-I., Mauk, B., and McIlwain, C. E.: Electron precipitation of evening diffuse aurora and its conjugate electron fluxes near the magnetospheric equator, J. Geophys. Res., 84, 2545, 1979.

Miller, K. L. and Vondrak, R. R.: A high-latitude phenomenological model of auroral precipitation and ionospheric effects Radio Sci., 20, 3, 431, 1985.

Østgaard, N., Stadsnes, J., Bjordal, J., Vondrak, R. R., Cummer, S. A., Chenette, D., Schulz, M., and Pronko, J.: Cause of the localized maximum of X-ray emission in the morning sector: A comparison with electron measurements, J. Geophys. Res., 105, 20869, 2000

Østgaard, N., Stadsnes, J., Bjordal, J., Germany, G. A., Vondrak, R. R., Parks, G. K., Cummer, S. A., Chenette,D., and Pronko, J.: Auroral electron distributions derived from combined UV and X-ray emissions, J. Geophys. Res., 106, 26 081, 2001.

Rees, M. H.: Auroral ionization and excitation by incident energetic electrons, Planet. Space. Sci., 11, 1209, 1963.

Rees, M. H., Lummerzheim, D., Roble, R. G., Winningham, J. D., Craven, J. D., and Frank, L. A.: Auroral energy deposition rate, characteristic electron energy, and ionospheric parameters de- rived from Dynamics Explorer 1 images, J. Geophys. Res., 93, 12, 841, 1988.

Rees, M. H.: Physics and Chemistry of the Upper Atmosphere, Cambridge Univ. Press, Cambridge, UK, 1989.

Robinson, R. M., Vondrak, R. R., Miller, K., Dabbs, T., and Hardy, D.: On calculating ionospheric conductances from the flux and energy of precipitating electrons, J. Geophys. Res., 92, 2565, 1987.

Spiro, R. W., Reiff, P. H., and Maher Jr., L. J.: Precipitating electron energy flux and auroral zone conductances - an empirical model, J. Geophys. Res., 87, 8215, 1982.

Thrane, E. V. and Piggott, W. R.: The collision frequency in the E- and D-regions of the ionosphere, J. Atmos. Terres. Phys., 28, $721,1966$.

Torr, M. R., Torr, D. G., Zukic, M., Johnson, R. B., Ajello, J., Banks, P., Clark, K., Cole, K., Keffer, C., Parks, G., Tsurutani, B., and Spann, J.: A far ultaviolet imager for the international solar-terrestrial physics mission, Space Sci. Rev., 71, 329, 1995.

Vickrey, J. F., Vondrak, R. R., and Matthews, S. J.: Energy depostion by energetic particles and Joule dissipation in the auroral ionosphere, J. Geophys. Res., 86, 65, 1981.

Vickrey, J. F., Vondrak, R. R., and Matthews, S. J.: The diurnal and latitudinal variation of auroral zone ionospheric conductivity, J. Geophys. Res., 87, 5148, 1982.

Vondrak, R. and Robinson, R.: Inference of high-latitude ionization and conductivity from AE-C measurements of auroral electron fluxes, J. Geophys. Res., 90, 7505, 1985.

Vondrak, R. R. and Baron, M. J.: Radar measurements of the latitudinal variation of auroral ionization, Radio Sci., 11, 11, 939, 1976.

Wallis, D. D. and Budzinski, E. E.: Empirical models of height integrated conductivities, J. Geophys. Res., 86, 125, 1981. 